\title{
Preface: Dedicated to the memory of Zoltán Ésik (1951-2016)
}

\author{
Manfred Droste ${ }^{1} \cdot$ Kim G. Larsen ${ }^{2}$
}

Published online: 23 January 2018

๑) Springer-Verlag GmbH Germany, part of Springer Nature 2018

This special issue contains papers on topics of the international workshop

Weighted Automata: Theory and Applications

(WATA 2014)

which took place at the Universität Leipzig, Germany, from May 5-9, 2014.

As for its predecessors WATA 2002, WATA 2004, WATA 2006, WATA 2008, WATA 2010 and WATA 2012, the goal of this workshop was to highlight the field of weighted automata, ranging from the theory of quantitative automata and logics to applications for real-time systems and natural language processing. The workshop was attended by 57 participants from 14 countries. Two tutorials were given by

Colin de la Higuera (Nantes, France),

Jacques Sakarovitch (Paris, France).

In addition, 9 invited lectures were presented by

Laurent Doyen (Cachan, France),

Zoltán Ésik (Szeged, Hungary),

Zoltán Fülöp (Szeged, Hungary)

Paul Gastin (Cachan, France),

Ricard Gavaldà (Barcelona, Spain),

Kim G. Larsen (Aalborg, Denmark),

Andreas Maletti (Stuttgart, Germany),

Eli Shamir (Jerusalem, Israel),

James Worrell (Oxford, Great Britain).
Furthermore, 29 talks were selected as contributed communications.

This workshop was financially supported by the DFG Research Training Group "Quantitative Logics and Automata" and by the Research Academy Leipzig.

After the workshop, a call for papers for a special issue of Soft Computing on "Quantitative Models and Weighted Automata" was issued. There were 15 submissions, and following the standard refereeing procedure, we were pleased to finally accept the present 7 papers for this special issue.

On May 25, 2016, our friend, colleague and co-editor of this special issue Zoltán Ésik passed away in Reykjavik, Iceland. Zoltán Ésik, Hungary's leading theoretical computer scientist, was a scientist of highest calibre. It is impossible to describe his many important achievements in a preface. For obituaries, we kindly refer the reader to a special issue of Acta Cybernetica, to the International Journal of Foundations of Computer Science, to the Journal of Automata, Languages and Combinatorics, to the Bulletin of the EATCS, and to the web page of Academia Europaea. Among many areas, Zoltán Ésik was very active in the field and supportive for the community of weighted automata, and we would like to dedicate this special issue to him.
Manfred Droste Kim G. Larsen

\footnotetext{
Manfred Droste

droste@informatik.uni-leipzig.de

Institut für Informatik, Universität Leipzig, Leipzig, Germany

2 Department of Computer Science, Aalborg University, Aalborg, Denmark
} 